\title{
FCC-ee: physics motivations
}

\author{
Christophe Grojean ${ }^{1,2, a}$ \\ ${ }^{1}$ Deutsches Elektronen-Synchrotron DESY, Notkestr. 85, 22607 Hamburg, Germany \\ 2 Humboldt-Universität zu Berlin, Institut für Physik, Newtonstr. 15, 12489 Berlin, Germany
}

Received: 14 May 2021 / Accepted: 7 August 2021

(C) The Author(s) 2022

\begin{abstract}
Learning from the first twelve years of LHC running, this essay offers a brief journey through the FCC-ee physics programme from refined precision measurements to probes of new physics, highlighting some of the commentaries between the different runs of FCC-ee at various energies as well as the synergies between the two FCC-ee and FCC-hh collider stages.
\end{abstract}

\section{The LHC legacy and the need for a change of HEP constitution}

The LHC has been a fantastic machine that confirmed the validity of the Standard Model (SM) of particle physics with great accuracy up to energies of several TeVs. The Higgs boson discovery in 2012 is a milestone in the history of physics, and it celebrates the triumph of the two pillars of twentieth-century physics, namely special relativity and quantum mechanics, combined in the realm of Quantum Field Theory [1]. The other major discovery of the LHC is the absence of New Physics at energy it was expected to show up, with two immediate consequences: traditional models of new physics are under siege and novel approaches named relaxation, Nnaturalness, etc., are being thought of with an interesting interplay between particle physics and cosmology. So, while the naturalness agents responsible for the stability of the weak scale under quantum corrections [2] might not explain the abundance of Dark Matter or the matter-antimatter imbalance, it was realised that the cosmological evolution of the Universe might have contributed to set the weak scale itself.

In these times of electoral fewer, one might remember that T. Jefferson himself acknowledged [3] that [he is] not an advocate for frequent changes in laws and Constitutions. But laws and institutions must go hand in hand with the progress of the human mind. As that becomes more developed, more enlightened, as new discoveries are made, new truths discovered and manners and opinions change, with the change of circumstances, institutions must advance also to keep pace with the times. With the LHC at hand, it might be the right moment to change what we mean by SM. The $\mathrm{SM}_{4}$, meant as the renormalisable $\mathrm{SU}(3)_{C} \times \mathrm{SU}(2)_{L} \times \mathrm{U}(1)_{Y}$ chiral gauge theory of three generations of quarks and leptons, is definitively a consistent theory (i.e. it is closed under radiative corrections and it has no pathology, except maybe a hypercharge Landau pole at very high energy). But we know for sure that it is not complete and it should be considered only as a low-energy Effective Field Theory (EFT). In this EFT, the traditional mass-dimension- 4 interactions are only the first, and most relevant at low energy, ones of

\footnotetext{
a e-mail: christophe.grojean@desy.de (corresponding author)
} 
a whole tower of interactions with more and more fields or more and more derivatives that constitute the full $\mathrm{SM}_{\infty}$. This new view brings new challenges and poses new questions: (1) what are the organising principles behind all these new interactions; (2) what are the actual symmetries of the $\mathrm{SM}_{\infty}$. For instance, if the energy scale suppressing the new interactions is not as high as $10^{16} \mathrm{GeV}$, the baryon and lepton number cannot be accidental symmetries as they are in $\mathrm{SM}_{4}$, but on the other hand quantum gravity probably forbids them to be exact global symmetries either. Similarly, other structural features of $\mathrm{SM}_{4}$, like the custodial symmetry or the GIM-FCNC structure of the flavour sector, call for further explanations.

We know for sure that new physics exists but there is no clear indication of the energy scale at which it will manifest: the smallness of flavour-changing neutral currents might indicate that this scale is as high as $10^{3} \mathrm{TeV}$ and the order of magnitude of the neutrino masses points to a scale of $10^{14} \mathrm{TeV}$, while the lower bound of the proton lifetime could be a sign that new physics will show up only at $10^{16} \mathrm{GeV}$ or above. But, at the same time, much lighter new physics could provide a very simple and elegant solution to mysterious puzzles like the fact that the strong interactions of QCD seem invariant under time-reversal (or more precisely under $\mathrm{CP}$ ), or like the quantum mechanically unbearable lightness of the elementary particles compared to the Planck mass, the natural scale of gravity, and even the ridiculously tiny value of the energy of the vacuum that fuels today acceleration of the expansion of the Universe. In the absence of indication of where to search, we need a broad, versatile and ambitious experimental programme that (1) will achieve legacy precision measurements and (2) can push the frontiers of the unknown. The FCC-ee+eh+hh programme combines these two aspects by providing more sensitivity, more precision and more energy than ever before.

\section{The need for more precision}

The benefit of more precision is threefold:

1. It will improve the indirect sensitivity to New Physics;

2. The precise values of the Higgs boson couplings will provide a better understanding of the structure of matter and of the Universe. For instance, the couplings to the light quarks will confirm if the Higgs field is indeed responsible for the up and down quarks masses that need to be delicately tuned to balance the electromagnetic contributions to the proton and neutron masses and therefore to ensure the stability of the nuclei. The electron Yukawa coupling that sets the electron mass ultimately controls the size of the atoms. The top quark Yukawa coupling decides, to a large extent, on the lifetime of the electroweak vacuum that the Universe settled in. The Higgs self-coupling controls the (thermo)dynamics of the EW phase transition that took place $10^{-10} \mathrm{~s}$ after the Big Bang and that could have resulted in the dominance of matter over anti-matter in the Universe today. The couplings of the Higgs to the $W$ and $Z$ gauge bosons set the lifetime of the Sun and all the stars (lifetime that turns out, a priori by accident, to be of the order of the typical timescale of biological evolution);

3. The values of the new interactions of the $\mathrm{SM}_{\infty}$ will also be helpful to reveal some "selection rules" intimately linked to new structures/symmetries. 
Table 1 Some operators relevant for Higgs physics and the impact of approximate symmetries on the estimated size of their coefficient [4]. Table taken from Ref. [5], see there for details

\begin{tabular}{|c|c|c|}
\hline Operator & $\begin{array}{l}\text { Naive (maximal) } \\
\text { scaling with } g_{*}\end{array}$ & $\begin{array}{l}\text { Symmetry/Selection Rule } \\
\text { and corresponding suppression }\end{array}$ \\
\hline$O_{y_{\psi}}=|H|^{2} \bar{\psi}_{L} H \psi_{R}$ & $g_{*}^{3}$ & Chiral: $y_{f} / g_{*}$ \\
\hline$O_{T}=\left(H^{\dagger} \stackrel{\leftrightarrow}{D}_{\mu} H\right)^{2}$ & $g_{*}^{2}$ & Custodial: $\left(g^{\prime} / g_{*}\right)^{2}, y_{t}^{2} / 16 \pi^{2}$ \\
\hline $\begin{array}{l}O_{G G}=|H|^{2} G_{\mu \nu}^{a} G^{a \mu \nu} \\
O_{B B}=|H|^{2} B_{\mu \nu} B^{\mu \nu}\end{array}$ & $g_{*}^{2}$ & $\begin{array}{l}\text { Shift symmetry: }\left(y_{t} / g_{*}\right)^{2} \\
\left.\text { Elementary Vectors: }\left(g_{s} / g_{*}\right)^{2} \text { (for } O_{G G}\right) \\
\left.\left(g^{\prime} / g_{*}\right)^{2} \text { (for } O_{B B}\right) \\
\text { Minimal Coupling: } g_{*}^{2} / 16 \pi^{2}\end{array}$ \\
\hline$O_{6}=|H|^{6}$ & $g_{*}^{4}$ & Shift symmetry: $\lambda / g_{*}^{2}$ \\
\hline$O_{H}=\left(\partial^{\mu}|H|^{2}\right)^{2}$ & $g_{*}^{2}$ & Coset Curvature: $\epsilon_{c}$ \\
\hline $\begin{array}{l}O_{B}=\left(H^{\dagger} \stackrel{\leftrightarrow}{D^{\mu}} H\right) \partial^{v} B_{\mu \nu} \\
O_{W}=\left(H^{\dagger} \sigma^{a} \stackrel{\leftrightarrow}{D^{\mu}} H\right) \partial^{v} W_{\mu \nu}^{a}\end{array}$ & $g_{*}$ & $\begin{array}{l}\text { Elementary Vectors: } g^{\prime} / g_{*}\left(\text { for } O_{B}\right) \\
g / g_{*}\left(\text { for } O_{W}\right)\end{array}$ \\
\hline $\begin{array}{l}O_{H B}=\left(D^{\mu} H^{\dagger} D^{v} H\right) B_{\mu \nu} \\
O_{H W}=\left(D^{\mu} H^{\dagger} \sigma^{a} D^{v} H\right) W_{\mu \nu}^{a}\end{array}$ & $g_{*}$ & $\begin{array}{l}\text { Elementary Vectors: } g^{\prime} / g_{*}\left(\text { for } O_{H B} \text { ) }\right. \\
g / g_{*}\left(\text { for } O_{H W}\right) \\
\text { Minimal Coupling: } g_{*}^{2} / 16 \pi^{2}\end{array}$ \\
\hline
\end{tabular}

This last point requires some further technical accounts. The $\mathrm{SM}_{\infty}$ Lagragian contains an infinite series of contact interactions among the SM particles:

$$
\mathcal{L}_{\infty}=\mathcal{L}_{4}+\sum_{d, i} \frac{c_{i}^{(d)}}{\lambda^{d-4}} \mathcal{O}_{i}^{(d)} .
$$

Simple dimensional arguments impose that the coefficient of each operator scale like

$$
c_{i}^{(d)} \sim(\text { coupling })^{n_{i}-2},
$$

where $n_{i}$ is the number of fields in the operator $\mathcal{O}_{i}^{(d)}$ and generically the coupling would be $g_{\star}$, the coupling of new physics to the SM particles. But there might exist several selection rules that lead to another scaling of the coefficients $c_{i}^{(d)}$, see Table 1 . That is how precise measurements of these coefficients could reveal the symmetry structure of $\mathrm{SM}_{\infty}$.

\section{Which machine?}

The choice for the future collider after the LHC is a delicate balance between physics return, technological challenges and feasibility, time scales for completion and exploitation as well as financial and political realities [6]. Each option has its pros and cons: (1) Hadron colliders have a (direct) larger mass reach but suffers from a signal-over-background ratio of order $10^{-10}$ that can be raised to $10^{-2} \div 10^{-1}$ after carefully triggering on specific characteristics of the expected signal events (thus leaving little room for discovery by serendipity). Furthermore, since hadrons are not the elementary actors of the hard processes, one only has a 
Table 2 FCC-ee run plan and anticipated number of relevant events produced [8]

\begin{tabular}{lllll}
\hline Phase & $\begin{array}{l}\text { Run duration } \\
\text { (years) }\end{array}$ & $\begin{array}{l}\text { Centre-of-mass } \\
\text { energies }(\mathrm{GeV})\end{array}$ & $\begin{array}{l}\text { Integrated } \\
\text { luminosity }\left(\mathrm{ab}^{-1}\right)\end{array}$ & Statistics \\
\hline FCC-ee-Z & 4 & $88-95$ & 150 & $5.10^{12} \mathrm{Z}$ \\
FCC-ee-W & 2 & $158-162$ & 12 & $10^{8} \mathrm{WW}$ \\
FCC-ee-H & 3 & 240 & 5 & $10^{6} \mathrm{ZH}$ \\
FCC-ee-tt & 5 & $345-365$ & 1.5 & $10^{6} t \bar{t}$ \\
\hline
\end{tabular}

statistical/PDF access to the characteristic energy of the events reconstructed in the detectors. (2) At lepton colliders, the signal-over-background ratio is easily of order unity and the beams can be polarised, giving an extra handle to enhance the dominant processes. Furthermore, the final states are easily identifiable in a clean environment, opening the door to the study of a wide range of channels that cannot be tagged at hadron colliders. (3) Circular colliders reach higher luminosity because the same particle bunches are used over many turns. Detectors can be installed at several interaction points while the same tunnel can be used for colliding first leptons and, a later stage, hadrons. Resonant transverse depolarisation allows for a precise beam-energy measurement. But the synchrotron radiation limits the energy available. (4) Linear colliders are easier to upgrade in energy and the beams are more easily polarised.

If exploration machines have always been at the heart of high-energy physics, the European Strategy Update for Particle Physics [7] prioritises an electron-positron Higgs factory as the best way to go the energy frontier. Table 2 specifies the foreseen run plan of the FCC-ee project. Superb statistics will be achieved in less than 15 years of operation.

\section{Electroweak programme at FCC-ee}

The high luminosity accumulated at the $Z$-pole (and the small associated systematics uncertainties in the measurements) is one of the strengths of the FCC-ee physics programme. Figure 1 nicely illustrates how these new EW measurements can be used to stress-test the $\mathrm{SM}_{4}$ predictions and to constrain some of its deformations. The improvement over what can be learnt from HL-LHC in the EW sector is spectacular. And the importance of improved EW measurements is at least threefold: (1) increased mass reach in indirect search for NP (e.g. a bound on the $S$ oblique parameter of order $10^{-2}$ probes Universal new physics up to a scale of $70 \mathrm{TeV}$ ), (2) reduced parametric uncertainties for other measurements and (3) reduced degeneracies in a global fit for the Higgs couplings.

\section{Higgs physics programme: Global Fit}

Within $\mathrm{SM}_{4}$, the Higgs couplings to all SM particles are uniquely fixed in terms of quantities already measured accurately (Fermi constant, masses and mixing angles). These predictions will be broken in $\mathrm{SM}_{\infty}$ and the measurements of the Higgs couplings through its production and decay rates give a way to directly probe the new higher-dimensional interactions. A global fit of the Higgs (and EW) data can be performed in a truly EFT analysis or in the socalled $\kappa$-parameter approach that focusses on particular deformations of the Higgs couplings 

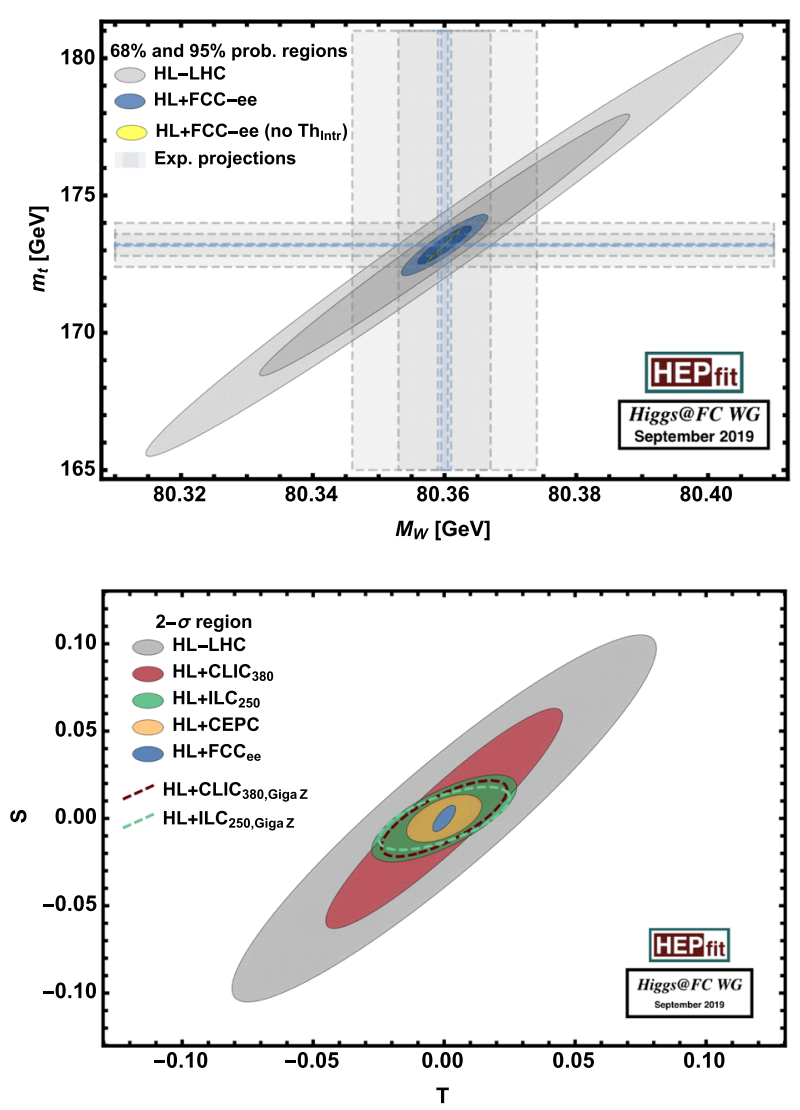

Fig. 1 Top: Interpretation of the EW measurements in $\mathrm{SM}_{4}$ that predicts the top mass a function of the $W$ mass and comparison with direct anticipated measurements. Bottom: Interpretations of the EW measurements in the $Z$-pole universal deformations of $\mathrm{SM}_{4}$ parameterised by the $S$ and $T$ oblique parameters. Plots taken from Ref. [9]

assuming no new Lorentz-structure. Table 3 reports the expected relative precision of the $\kappa$ parameters from the FCC measurements alone and in combination with HL-LHC. One can notice an important synergy in all the statistically limited channels, $\gamma \gamma, Z \gamma$ and $\mu \mu$, but also in the determination of the top Yukawa (for which HL-LHC overcomes the absence of run at the $t \bar{t} h$ threshold at FCC-ee) and conversely the ability to identify the charm quarks in the FCC-ee detectors mitigates the absence of efficient charm-tagging algorithm at HL-LHC.

Table 3 also gives the sensitivity of the Higgs coupling measurements from the integrated FCC-ee/eh/hh programme. These impressive numbers result from a remarkable synergy between the lepton and hadron runs. One can give three significant examples. The first concerns the Higgs decay: while FCC-hh could, on its own, measure the Higgs invisible branching ratio, it could say close to nothing on its untagged branching ratio that requires a lepton machine to identify Higgs events produced in association with a $Z$ boson independently of the Higgs decay modes thanks to the measurement of the $Z$ recoil mass. This gives a unique access to the Higgs width and to the absolute normalisation of the Higgs couplings and it can then be interpreted as a bound on exotic decay channels. The second example of ee-hh synergy deals with the measurement of the top Yukawa coupling: FCC-hh is determining 


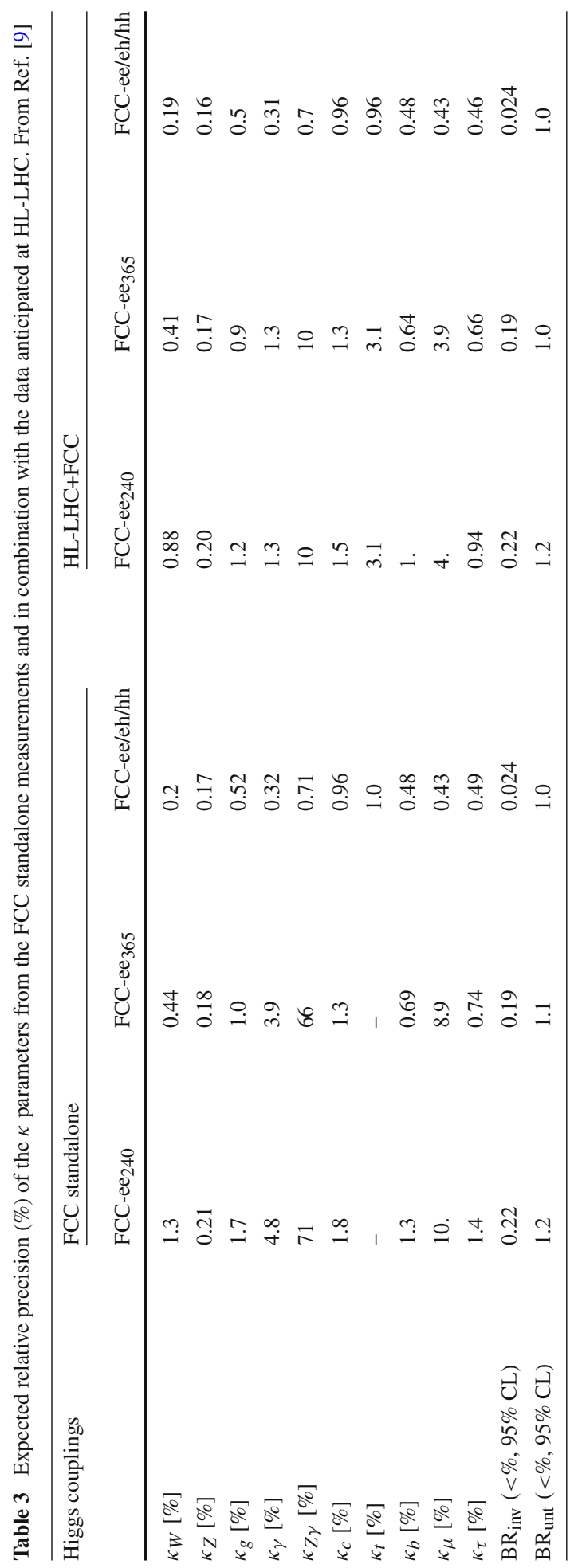


Fig. 2 Top: 68 and 95\% CL determination of the top Yukawa coupling at FCC-hh with and without the input of the top- $Z$ coupling from FCC-ee data. Bottom: 68 and $95 \% \mathrm{CL}$ determination of the Higgs self-coupling coupling at FCC-hh with and without the input of FCC-ee data. Plots prepared by J. de Blas [10]
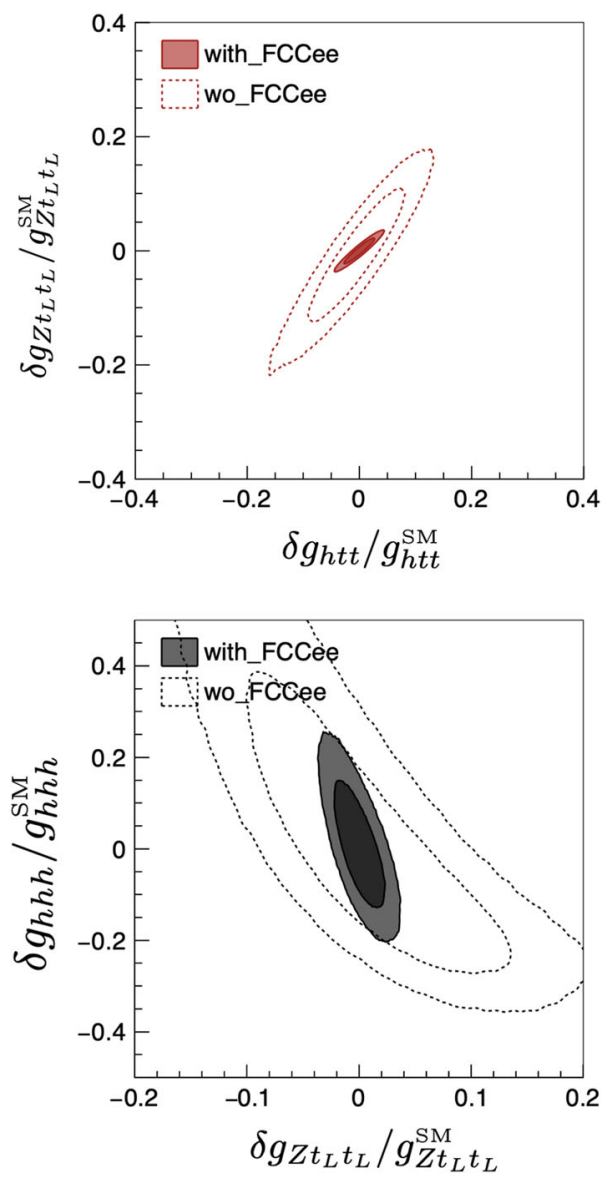

this coupling through the ratio $t \bar{t} h / t \bar{t} Z$ in which the uncertainties drop to the level of $1 \%$ instead of $10 \%$ or so in the individual measurements of $\sigma(t \bar{t} h)$ and $\sigma(t \bar{t} Z)$. To extract the top Yukawa coupling itself, the precise knowledge of the top EW couplings from FCC-ee measurement is paramount. Figure 2 beautifully illustrates this synergy. The right plot in this figure further shows how FCC-ee is (indirectly) needed to reach a 5\% sensitivity in the determination of the Higgs trilinear coupling at FCC-hh extracted from the measurement of the gluon-fusion double Higgs production cross-section, highly sensitive to the top Yukawa in addition of the Higgs self-coupling.

The Higgs programme at FCC-ee also benefits a lot from measurements performed at the Z-pole as illustrated in Fig. 3. The electroweak measurements performed at LEP would indeed be a limiting factor in the extraction of the Higgs couplings, while the new $Z$ data at FCC-ee can bring a $50 \%$ improvement in the determination of some Higgs couplings compared to a fit relying on the old LEP EW measurements only. This improvement comes both directly from the improved reach on the $h Z e e$ contact interactions and indirectly from the improvement on the aTGCs which are related to the $h Z Z$ and $h W W$ anomalous couplings. 
Fig. 3 A scheme-ball illustration of the correlations between Higgs and EW sector couplings. The outer bars give the one-sigma precision on the individual coupling with (bottom) and without (top) a dedicated $Z$-pole run at FCC-ee and CEPC. The open white dots is the projected sensitivity in the limit of infinite precision in EW measurements. The $10^{12} Z$ 's expected at FCC-ee almost saturates this limit. Adapted from Ref. [11]

\section{Higgs self-coupling}
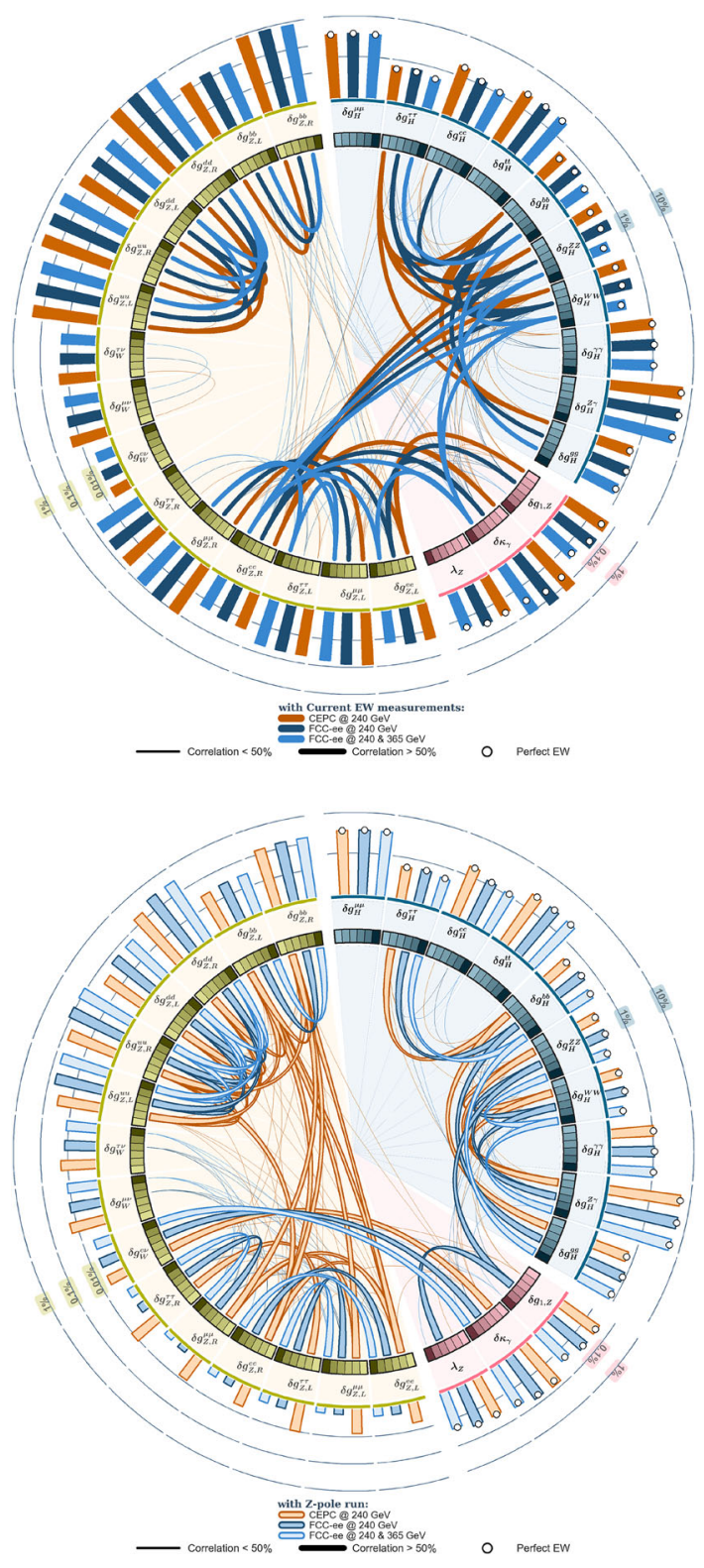

The measurement of the Higgs self-interactions, and more generally the reconstruction of the Higgs potential, is of very high priority in the Higgs physics programme (at HL-LHC and at any future colliders). Unfortunately, these self-interactions, beyond the simple kinematical 2-point interaction that corresponds to the Higgs boson mass, are not proper observables and they need to be inferred for production or decay rates that show a dependence on the values of these Higgs self-interactions. Traditionally, the Higgs cubic self-coupling is extracted from the measurement of double Higgs boson production either by gluon fusion at hadron colliders or in association with a $Z$ boson at lepton colliders. This would require to reach 
an energy threshold high enough, which is surely an option for a linear $e^{+} e^{-}$collider but is currently not foreseen at a circular $e^{+} e^{-}$collider. Still, low-energy colliders can probe the Higgs cubic self-coupling by exploiting loop corrections to single Higgs channels that can be measured to a very high precision. This approach, first proposed in Ref. [12], indeed allows for set stringent bound on the Higgs trilinear interaction, which can easily surpass the HL-LHC one. However, for this bound to be robust, one must be able to disentangle the different new-physics effects that may affect simultaneously the single Higgs rates. It was realised [13] that, to isolate the Higgs self-coupling contribution, it is essential to compare the $Z h$ cross section at two sufficiently different energies, e.g. $240 \mathrm{GeV}$ and $350 \mathrm{GeV}$, still below the $500 \mathrm{GeV} Z h h$ threshold, see Fig. 4. With the baseline FCC-ee run plan, a precision of $42 \%$ can be achieved on the determination of $\delta \kappa_{\lambda}$, reduced to $33 \%$ in combination with HL-LHC. In case 4 IPs are considered, and a reorganisation of the run plan to accumulate $12 / \mathrm{ab}$ at $240 \mathrm{GeV}, 5.5 / \mathrm{ab}$ at $350 / 365 \mathrm{GeV}$, the sensitivity could even reach $24 \%$ [9].

\section{7 s-channel Higgs production and electron Yukawa coupling}

LHC has established that the Higgs mechanism is indeed responsible for the masses of the $W$ and $Z$ gauge bosons and of the heavy quarks and leptons. Whether this is also the case for the masses of the light fermions that the matter surrounding us is made of is still a largely open question. FCC-ee can bring a definitive answer for what concerns the electrons as, in a dedicated run potentially accumulating a luminosity of 20/ab at a centre-of-mass energy $\sqrt{s}=125 \mathrm{GeV}$ with a monochromatisation of the beams of order $10 \mathrm{MeV}$, enough statistics will be accumulated to observe the resonant Higgs production in s-channel, see Fig. 5.

Surely interesting on its own, the determination of the electron Yukawa coupling is also important in the interpretation of the bounds of electric dipole moment (EDM) of the electron to constrain possible $\mathrm{CP}$ violation sourced by the $\mathrm{SM}_{\infty}$ contact interactions. In that sense, the electron Yukawa is a bridge between the low energy and high- $p_{T}$ worlds and FCC-ee is the key to open this bridge. The expected improvement by 3 orders of magnitude on the eEDM bound [15] could then offer a sensitivity to interactions with a characteristic scale of order $1000 \mathrm{TeV}$.

\section{Other aspects of the FCC-ee physics programme}

\subsection{Flavour physics}

As stressed in the FCC CDR [8], the $10^{12} \mathrm{Z}$ decays to be delivered by FCC-ee can be exploited to further enrich the knowledge of flavour physics, beyond what will emerge by the start of the FCC-ee program from the upgraded LHCb and Belle II experiments. Some examples of particularly interesting channels to study are:

- the decays $B_{s} \rightarrow \tau^{+} \tau^{-}$and $\bar{B}^{0} \rightarrow K^{\star 0}(892) \tau^{+} \tau^{-}$that will bring complementary information to the $b \rightarrow s \ell^{+} \ell^{-}$transitions scrutinised at LCHb.

- the search for lepton flavour violating decays of the $Z$ boson, e.g. $Z \rightarrow e \mu, \mu \tau$ or $e \tau$, and of the $\tau$, e.g. $\tau \rightarrow 3 \mu$ and $\tau \rightarrow \mu \gamma$, that would provide indisputable evidence for BSM physics.

- the leptonic decays of the $\tau, \tau \rightarrow \mu \nu \nu$ and $\tau \rightarrow e \nu \nu$ that will allow for significantly improved tests of lepton flavour universality challenged by current LHCb measurements. 
Fig. 4 Top: example of one-loop diagrams involving the trilinear Higgs coupling contributing to $e^{+} e^{-} \rightarrow Z h$ production. Bottom: One-sigma bound on $\delta \kappa_{\lambda}$, the deviation of the Higgs cubic self-coupling, relative to its SM values, obtained from single Higgs measurement at lepton colliders as a function of the integrated luminosity collected at both 240 and $350 \mathrm{GeV}$.

Conservative (solid) and optimistic (dashed) assumptions are used for the precision of diboson measurements. From Ref. [13]
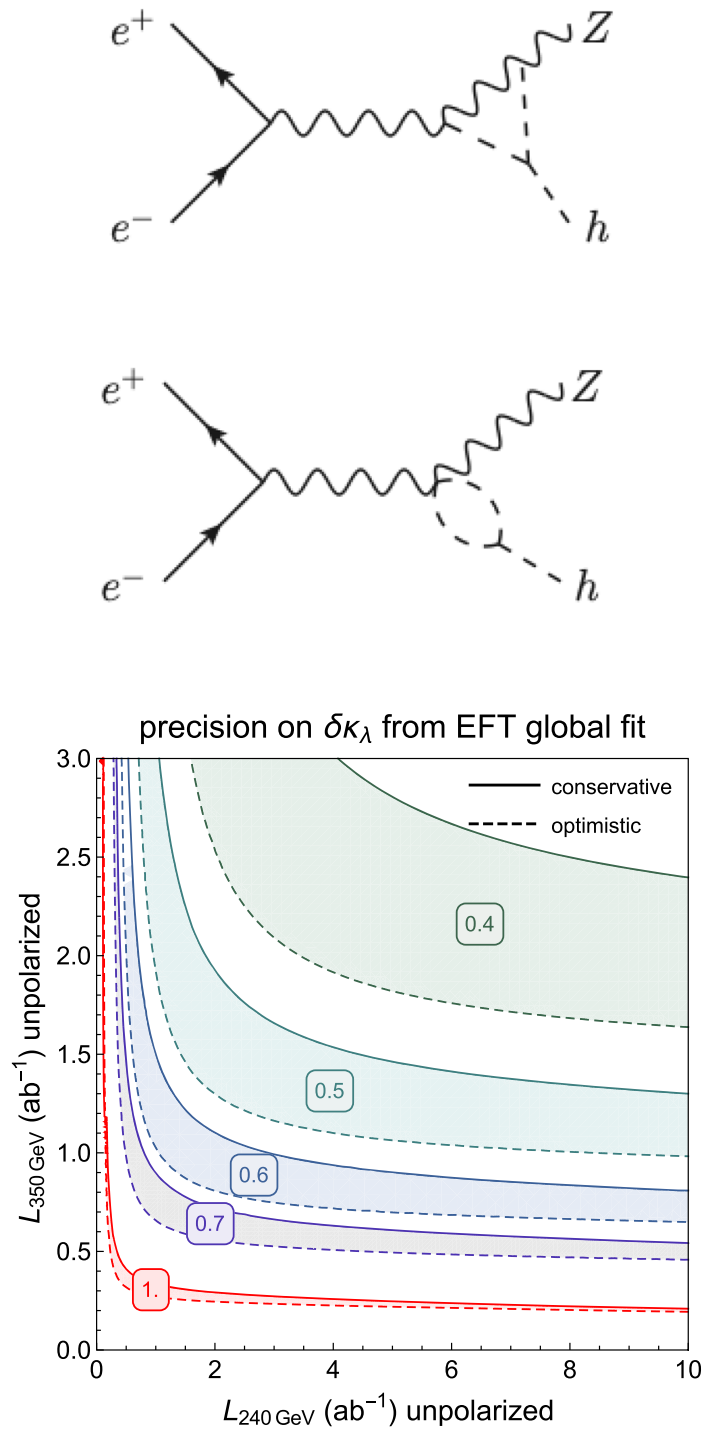

- $\Delta F=2$ quark transitions, e.g. in $B_{d}$ and $B_{s}$ meson oscillations that will improve the determination of the less-precisely-known parameters of the CKM matrix.

It should also be mentioned that FCC-ee could probe scenarios of new physics with light and weakly coupled new physics. Particularly, motivated examples are models with axion-like particles that could be observed via their mixing with SM mesons in processes like $K_{L} \rightarrow \pi^{0} a \rightarrow \pi^{0} \gamma \gamma$ or $K^{+} \rightarrow \pi^{+} a \rightarrow \pi^{+} \gamma \gamma$ or that can be produced directly in association with a photon, $e^{+} e^{-} \rightarrow \gamma a$, or with a Higgs boson, $e^{+} e^{-} \rightarrow h a$. These processes are probing ALPs in the mass range between $10 \mathrm{MeV}$ to a few 10 's of GeV [16]. 
Fig. 5 Resonant s-channel Higgs production (top) and the corresponding projected sensitivity in the determination of electron Yukawa coupling at FCC-ee in two different dedicated configurations (one year with two baseline detectors or three years with four detectors) and comparison with current and future planned colliders (bottom). Plot taken from Ref. [14], see there for details

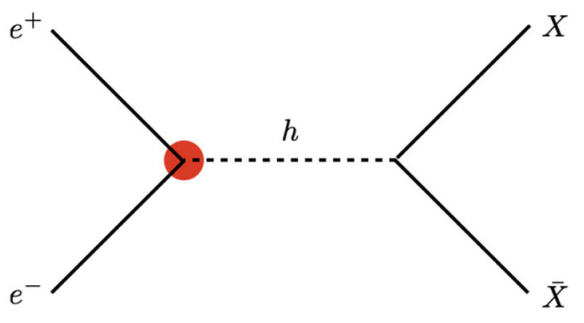

Upper Limits / Precision on $\kappa_{\mathrm{e}}$

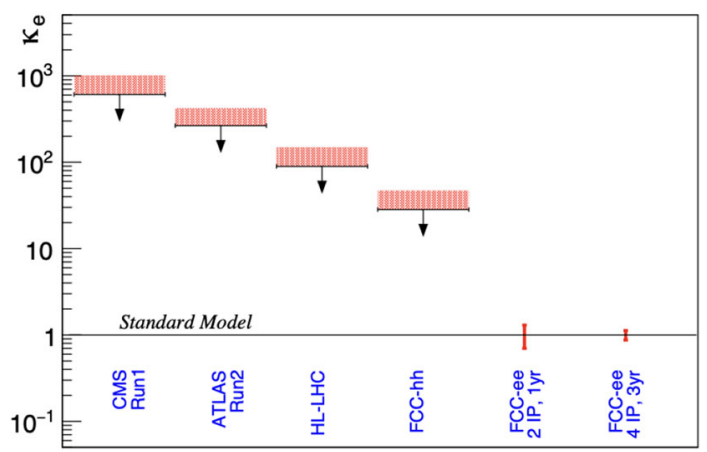

\subsection{Dark sector exploration}

Dark Matter could be part of a whole Dark Sector with complex interactions and involved spectrum as already accounted in the visible SM sector. The neutrality and spinless nature of the Higgs boson can make easily it a portal between the SM and the Dark Sector. The FCC-ee sensitivity to the invisible decays the Higgs boson and of the $Z$ boson offers a new way to probe these portal models. And, in addition, exotic decays of the Higgs that FCCee will have access to could also help revealing dark showers resulting from fragmentation and hadronisation processes in the dark sector. Thanks for their clean environment, lepton colliders like FCC-ee will be particularly good in identifying exotic decay channels with hadronic final states and/or missing energy, see Fig. 6. Dedicated beam-dump detectors might even further improve the sensitivity to scenarios featuring long lived particles. Though these searches, FCC-ee will nicely complement the quest for Dark Matter in scenarios away from the standard paradigm of weakly interacting massive particles that will be thoroughly probed directly at the FCC-hh stage.

\subsection{Neutrino physics}

While neutrino masses can be easily accommodated by dimension- 5 operators of $\mathrm{SM}_{\infty}$, there is no guarantee that this is the way Nature has chosen. FCC-ee will probe the existence of neutrino mass partners that, though totally neutral to all the SM gauge interactions, could acquire a coupling to the electroweak bosons via a mixing to the ordinary neutrinos. Their existence can be revealed indirectly, via their contribution to the muon lifetime $\mu \rightarrow e v_{\mu} \bar{v}_{e}$ that serves a key input to electroweak precision measurements, or more directly in decays of the $Z$ bosons, $Z \rightarrow v N$, with spectacular and clean signatures of displaced vertices when 


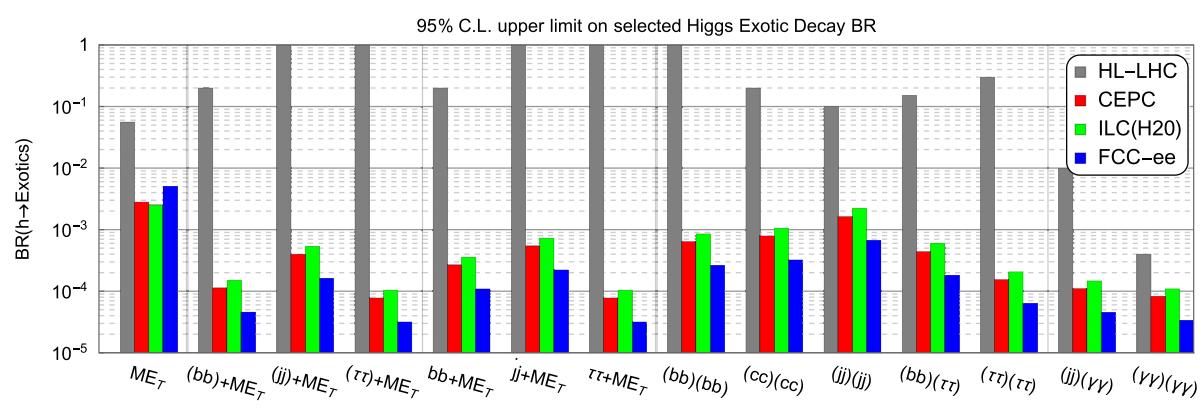

Fig. $695 \%$ CL upper limit on selected Higgs exotic decay branching fractions at HL-LHC, CEPC, ILC and FCC-ee. Plot taken from Ref. [17], see there for details

$N$ is sufficiently long-lived as expected in models of leptogenesis aiming at dynamically generating the baryon asymmetry of the Universe. See Ref. [8], and references therein, for a more comprehensive discussion of the FCC-ee potential in neutrino physics.

\section{Conclusion}

A circular Higgs factory like FCC-ee has a rich potential characterised by (1) some legacy measurements that will go into the physics textbooks and (2) several refinements in our understanding of Nature addressing long-standing questions like the nature of the EW phase transition and the naturalness of the weak scale. It is an essential part of an integrated programme to probe directly the energy frontier with a circular hadronic machine. While its predecessor colliders, SPS/LEP/Tevatron/LHC, have been establishing the foundations of $\mathrm{SM}_{4}$, FCC-ee will be the machine to explore the full $\mathrm{SM}_{\infty}$ and to reveal its structures/symmetries that will hint to its interactions with new degrees of freedom needed to explain the Universe as revealed from cosmological observations. As illustrated with the example of electron Yukawa coupling and its contribution to the eEDM, FCC is also essential to make use of a worldwide complementary and vibrant diversity programme beyond colliders.

Acknowledgements I acknowledge support by the Deutsche Forschungsgemeinschaft under Germany's Excellence Strategy EXC 2121 "Quantum Universe" - 390833306. And the Future Circular Collider Innovation Study (FCCIS) project has received funding from the European Union's Horizon 2020 research and innovation programme under grant No 951754. I am particularly grateful to J. De Blas, G. Durieux, J. Gu, A. Paul and the members of the ECFA Higgs@FC working group for insightful collaborations.

Funding Open Access funding enabled and organized by Projekt DEAL. This project is co-funded from the European Union's Horizon 2020 research and innovation programme under grant agreement No 95175.

Open Access This article is licensed under a Creative Commons Attribution 4.0 International License, which permits use, sharing, adaptation, distribution and reproduction in any medium or format, as long as you give appropriate credit to the original author(s) and the source, provide a link to the Creative Commons licence, and indicate if changes were made. The images or other third party material in this article are included in the article's Creative Commons licence, unless indicated otherwise in a credit line to the material. If material is not included in the article's Creative Commons licence and your intended use is not permitted by statutory regulation or exceeds the permitted use, you will need to obtain permission directly from the copyright holder. To view a copy of this licence, visit http://creativecommons.org/licenses/by/4.0/. 


\section{References}

1. Y. Nir, CERN Cour. 60, 41-45 (2020). [arXiv:2010.13126 [hep-ph]]

2. G. F. Giudice, [arXiv: 1710.07663 [physics.hist-ph]]

3. T. Jefferson, https://is.gd/v3uxCe

4. G. F. Giudice, C. Grojean, A. Pomarol and R. Rattazzi, JHEP 06 (2007), 045 [arXiv:hep-ph/0703164 [hep-ph]]

5. R. Contino, A. Falkowski, F. Goertz, C. Grojean, F. Riva, JHEP 07, 144 (2016). [arXiv:1604.06444 [hep-ph]]

6. M. Mangano, PoS TASI2018 (2019), 008 [arXiv:1905.07489 [hep-ph]]

7. 2020 Update of the European Strategy for Particle Physics, https://is.gd/4T12oh

8. A. Abada et al., FCC. Eur. Phys. J. C 79(6), 474 (2019)

9. J. de Blas, M. Cepeda, J. D-Hondt, R.K. Ellis, C. Grojean, B. Heinemann, F. Maltoni, A. Nisati, E. Petit, R. Rattazzi et al., JHEP 01, 139 (2020). [arXiv:1905.03764 [hep-ph]]

10. J. De Blas, talk at FCC-CDR symposium, CERN, 2019 https://is.gd/V31dDG

11. J. De Blas, G. Durieux, C. Grojean, J. Gu, A. Paul, JHEP 12, 117 (2019). [arXiv:1907.04311 [hep-ph]]

12. M. McCullough, Phys. Rev. D 90 (2014) no.1, 015001 [erratum: Phys. Rev. D 92 (2015) no.3, 039903] [arXiv:1312.3322 [hep-ph]]

13. S. Di Vita, G. Durieux, C. Grojean, J. Gu, Z. Liu, G. Panico, M. Riembau, T. Vantalon, JHEP 02, 178 (2018). [arXiv:1711.03978 [hep-ph]]

14. A. Blondel and P. Janot, [arXiv:1912.11871 [hep-ex]]

15. N. R. Hutzler, A. Borschevsky, D. Budker, D. DeMille, V. V. Flambaum, G. Gabrielse, R. F. Garcia Ruiz, A. M. Jayich, L. A. Orozco and M. Ramsey-Musolf, et al. [arXiv:2010.08709 [hep-ph]]

16. M. Bauer, M. Heiles, M. Neubert and A. Thamm, Eur. Phys. J. C 79 (2019) no.1, 74 [arXiv:1808.10323 [hep-ph]]

17. Z. Liu, L. T. Wang and H. Zhang, Chin. Phys. C 41 (2017) no.6, 063102 [arXiv:1612.09284 [hep-ph]] 